\title{
Exclusive hadronization in $\gamma^{*} \gamma \rightarrow \pi \pi^{*}$
}

\author{
M. Diehl ${ }^{\mathrm{a}}$, T. Gousset ${ }^{\mathrm{b}}$ and B. Pire ${ }^{\mathrm{c}}$. \\ a Deutsches Elekronen-Synchroton DESY, 22603 Hamburg, Germany \\ ${ }^{\text {b}}$ Subatech, B.P. 20722, 44307 Nantes, France \\ ${ }^{\mathrm{c}}$ Centre de Physique Théorique, École Polytechnique, 91128 Palaiseau, France
}

We perform a QCD analysis of exclusive two-meson production in $\gamma^{*} \gamma$-collisions in the kinematical domain of large photon virtuality $Q^{2}$ and small hadronic invariant mass $W^{2}$. In these kinematics the amplitude factorizes into a perturbative subprocess $\gamma^{*} \gamma \rightarrow q \bar{q}$ and a two-meson distribution amplitude. This opens the way to study new hadronic matrix elements related with the physics of hadronization and confinement.

\section{INTRODUCTION}

The physics of the reaction $\gamma^{*} \gamma \rightarrow \pi \pi$ in the kinematical domain where the photon virtuality is large compared to the hadronic invariant mass has recently been shown to be very interesting from a QCD point of view (棈), see also the pioneering work by D. Müller et al. [2]). The reason is that this process factorizes [3] into a perturbatively calculable, short-distance dominated subprocess $\gamma^{*} \gamma \rightarrow q \bar{q}$ and a non-perturbative QCD matrix element we have called generalized distribution amplitude (GDA). In fact, this generalizes the factorization of the $\gamma-\pi$ transition form factor measured in $\gamma^{*} \gamma \rightarrow \pi^{0}$, which has been extensively studied theoretically and experimentally 4.5 .

Note that $\gamma^{*} \gamma \rightarrow \pi \pi$ is related by crossing to deep virtual Compton scattering on a meson, $\gamma^{*} \pi \rightarrow \gamma \pi$, which at large $Q^{2}$ and small squared momentum transfer between the hadrons factorizes into a hard photon-parton scattering and a skewed (i.e. off-forward, non-forward, or nondiagonal) parton distribution [6]. The $\gamma^{*} \gamma$ and Compton processes share many features, in particular their scaling behavior in $Q^{2}$ and a helicity selection rule for the virtual photon.

\footnotetext{
*Talk given at the International Conference on the Structure and Interactions of the Photon (PHOTON 99), Freiburg im Breisgau, Germany, 23-27 May 1999, to appear in Nucl. Phys. B (Proc. Suppl.)
}

\section{KINEMATICS}

The reaction we are interested in is either

$e(k)+e(l) \rightarrow e\left(k^{\prime}\right)+e\left(l^{\prime}\right)+\pi^{a}(p)+\pi^{b}\left(p^{\prime}\right)$

or

$e(k)+\gamma\left(q^{\prime}\right) \rightarrow e\left(k^{\prime}\right)+\pi^{a}(p)+\pi^{b}\left(p^{\prime}\right)$,

where $a, b=+, 0,-$ and

$$
\begin{aligned}
q & =k-k^{\prime}, \quad Q^{2}=-q^{2}, \\
q^{\prime} & =l-l^{\prime}, \quad q^{\prime 2} \rightarrow 0, \\
P & =p+p^{\prime}, \quad W^{2}=P^{2}, \\
s_{e e} & =(k+l)^{2}, \quad s_{e \gamma}=\left(k+q^{\prime}\right)^{2} .
\end{aligned}
$$

In the $\gamma^{*} \gamma$ center-of-mass frame, choosing the $z$ axis along $\mathbf{q}$, natural variables are $\theta$, the polar angle of $\mathbf{p}$, and $\varphi$, the angle between the leptonic plane and the hadronic plane. To completely specify the kinematics one needs three further variables, which can be chosen as $Q^{2}, W^{2}$ and $y=\left(W^{2}+Q^{2}\right) / s_{e \gamma}$.

We shall also use the variable $\zeta$, defined as the light-cone fraction of momentum carried by $\pi^{a}(p)$ with respect to the pion pair. It is related to $\theta$ through

$$
\zeta=\frac{1}{2}\left(1+\sqrt{1-\frac{4 m_{\pi}^{2}}{W^{2}}} \cos \theta\right) .
$$




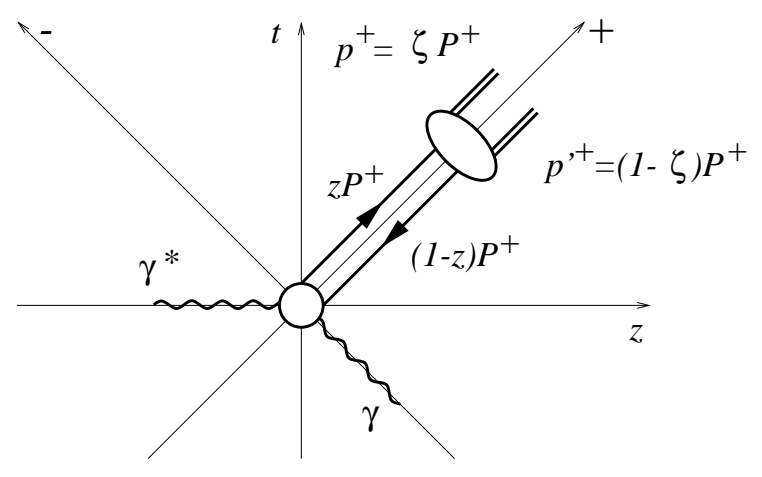

Figure 1. Spacetime diagram in the Breit frame, obtained by boosting from the center of mass along $z$.

\section{FACTORIZATION}

The spacetime cartoon of the process one can derive from power counting and factorization arguments is shown in Fig. 1.

In the Breit frame the real photon moves fast along $-z$ and is scattered into an energetic hadronic system moving along $+z$. The hard part of this process takes place at the level of elementary constituents, and the minimal number of quarks and gluons compatible with conservation laws (color etc.) emerge. At Born level one has simply $\gamma^{*} \gamma \rightarrow q \bar{q}$, but through a quark box the photons can also couple to two gluons. Each quark or gluon carries a fraction $z$ or $1-z$ of the large light-cone momentum component $P^{+}$. Subsequently the soft part of the reaction, i.e. hadronization into a pion pair, takes place.

At leading order in $\alpha_{S}$ one has for the hadronic tensor

$$
\begin{aligned}
T^{\mu \nu} & =i \int d^{4} x e^{-i q \cdot x}\left\langle\pi \pi\left|T J_{\mathrm{em}}^{\mu}(x) J_{\mathrm{em}}^{\nu}(0)\right| 0\right\rangle \\
& =-g_{T}^{\mu \nu} \sum_{q} \frac{e_{q}^{2}}{2} \int_{0}^{1} d z \frac{2 z-1}{z(1-z)} \Phi_{q}
\end{aligned}
$$

where $g_{T}^{\mu \nu}$ denotes the metric tensor in transverse space. Here we introduced for each quark flavor the generalized distribution amplitude

$$
\Phi_{q}=\int \frac{d x^{-}}{2 \pi} e^{-i z\left(P^{+} x^{-}\right)}\left\langle\pi \pi\left|\bar{q}\left(x^{-}\right) \gamma^{+} q(0)\right| 0\right\rangle,
$$

in light cone gauge $A^{+}=0 . \Phi_{q}$ depends on the quark light-cone fraction $z$, on the kinematical variables $\zeta$ and $W^{2}$ of the pions, and on its factorization scale.

Contracting with the photon polarization vectors we see that in order to give a nonzero $\gamma^{*} \gamma \rightarrow$ $\pi \pi$ amplitude the virtual photon must have the same helicity as the real one, in particular it must be transverse. As in the case of virtual Compton scattering this is a direct consequence of chiral invariance in the collinear hard scattering process [7]. We also find that the $\gamma^{*} \gamma$ amplitude is independent of $Q^{2}$ at fixed $\zeta$ and $W^{2}$, up to logarithmic scaling violation to be discussed later.

There will of course be power corrections in $1 / Q$ to this leading mechanism. An example is the hadronic component of the real photon, which might be modeled by vector meson dominance. From power counting arguments one obtains that this contribution is suppressed, as it is in virtual Compton scattering. Our process can also be treated within the operator product expansion, which allows for a systematic analysis of higher twist effects.

\section{RELATION WITH THE PHOTON STRUCTURE FUNCTIONS}

The exclusive process we consider here may be seen as the limit of the inclusive reaction $\gamma^{*} \gamma \rightarrow X$ [8], when unlike in the Bjorken limit the hadronic mass squared $W^{2}$ is not scaled up with $Q^{2}$ but remains fixed and small enough for the final state $X$ to be saturated by the $\pi \pi$ state. Let us examine the connection to some aspects of the photon structure functions, pointing out the specificity of the present kinematical limit.

The differential unpolarized cross section for the reaction $e+\gamma \rightarrow e+X$ is

$$
\begin{aligned}
& \frac{d \sigma}{d x_{B} d Q^{2}}=\frac{4 \pi \alpha^{2}}{x_{B} Q^{4}} \\
& \quad \times\left\{\left[1+(1-y)^{2}\right] x_{B} F_{T}^{\gamma}+(1-y) F_{L}^{\gamma}\right\},
\end{aligned}
$$


where in addition to the kinematical notation of Section 2 we introduced $x_{B}=Q^{2} /\left(Q^{2}+W^{2}\right)$. $\alpha$ is the QED coupling, and $F_{T}^{\gamma}$ and $F_{L}^{\gamma}$ are the photon structure functions, the index indicating the polarization of the virtual photon. Their connection to the other familiar set, $F_{1}$ and $F_{2}$, is given by $F_{1}=F_{T}$ and $F_{2}=2 x_{B} F_{T}+F_{L}$.

The pointlike contribution of a light quark with charge $e_{q}$ to $F_{T}^{\gamma}$ and $F_{L}^{\gamma}$, computed at zeroth order in the strong coupling is

$$
\begin{gathered}
F_{T}^{\gamma}=\frac{3 \alpha}{2 \pi} e_{q}^{4}\left\{\left[x_{B}^{2}+\left(1-x_{B}\right)^{2}\right] \ln \frac{\left(1-x_{B}\right) Q^{2}}{x_{B} m_{q}^{2}}\right. \\
\left.-\left(2 x_{B}-1\right)^{2}\right\}, \\
F_{L}^{\gamma}=\frac{3 \alpha}{2 \pi} e_{q}^{4}\left\{8 x_{B}^{2}\left(1-x_{B}\right)\right\},
\end{gathered}
$$

in the Bjorken limit, where $Q^{2}, W^{2} \gg m_{q}^{2}$. Note that $m_{q}$ is to be understood here as an effective mass that regulates the divergence in the box diagram. We see that in the region of fixed $W^{2}$ with $Q^{2} \gg W^{2}$ (where $1-x_{B} \sim W^{2} / Q^{2}$ ), these functions are

$$
\begin{aligned}
& F_{T}^{\gamma} \sim \frac{3 \alpha}{2 \pi} e_{q}^{4}\left\{\ln \frac{W^{2}}{m_{q}^{2}}-1\right\} \\
& F_{L}^{\gamma}=O\left(\frac{W^{2}}{Q^{2}}\right)
\end{aligned}
$$

which shows the disappearance of the $\ln Q^{2}$ behavior in $F_{T}^{\gamma}$ and the vanishing of $F_{L}^{\gamma}$ as expected.

Let us also stress that the hadronic part of the photon structure function (usually parameterized by a vector dominance ansatz), which in general is only suppressed by a factor $\ln Q^{2}$ with respect to the pointlike part, does not survive our particular limiting procedure. As already noted it becomes a $O(1 / Q)$ correction.

\section{GENERALIZED DISTRIBUTION AMPLITUDES.}

The physics contained in generalized distribution amplitudes goes beyond that of a $q \bar{q}$ distribution amplitude of a meson: since two hadrons are formed $\Phi$ does not select their lowest Fock states; in this respect it is related to ordinary parton distributions and to fragmentation functions. If, however, $W$ is at or near the mass of a resonance with appropriate quantum numbers, such as an $f_{0}$, it will contain physics of the distribution amplitude for the resonance and of its decay into two pions [9].

The same crossing procedure that connects our $\gamma^{*} \gamma$ process with deep virtual Compton scattering allows one to relate generalized distribution amplitudes to skewed parton distributions by an analytic continuation in the invariant mass variable $W^{2} 10$.

Whereas time reversal invariance constrains ordinary distribution amplitudes and parton distributions to be real valued functions up to convention dependent phases, our generalized distributions are complex. We notice in (5) that the hard scattering kernel at Born level is purely real so that the imaginary part of the $\gamma^{*} \gamma$ amplitude is due to $\operatorname{Im} \Phi$; it corresponds to rescattering and resonance formation in the soft transition from the partons to the final state hadron pair. In fact, Watson's theorem allows to deduce the phase of $\Phi\left(z, \zeta, W^{2}\right)$ from the phase shift analysis of $\pi \pi$ scattering for $W$ not too large [9].

Up to now we have discussed generalized $q \bar{q}$ distribution amplitudes. Beyond tree level in the hard scattering the hadron pair can however also originate from two gluons. Since gluons are known to be important in fragmentation and in parton distributions, one can expect the generalized $g g$ distribution to be of the same order as the one for $q \bar{q}$.

QCD radiative corrections to the hard scattering will as usual lead to logarithmic scaling violation. At this point it is useful to remember the analogy of our process with the transition $\gamma^{*} \gamma \rightarrow \pi^{0}$. The evolution can be obtained from the hard scattering kernel alone and remains the same if we replace $\Phi$ with the distribution amplitude of a meson with the appropriate quantum numbers, say an $f_{0}$. The generalized distribution amplitudes thus follow the usual EfremovRadyushkin-Brodsky-Lepage evolution [1] for a meson. Notice the peculiarity of the present channel that generalized $q \bar{q}$ and $g g$ distributions mix under evolution [12]. 


\section{PHENOMENOLOGY}

\subsection{The Bremsstrahlung subprocess}

In $e \gamma$ collisions the process $\gamma^{*} \gamma \rightarrow h \bar{h}$ we want to study competes with bremsstrahlung, where the hadron pair $h \bar{h}$ originates from a virtual photon radiated off the lepton [13]. This process produces the pair in the $C$-odd channel and hence does not contribute for $h=\bar{h}$, in particular not for $h=\pi^{0}$. Its amplitude can be computed from the value of the timelike elastic form factor measured in $e^{+} e^{-} \rightarrow h \bar{h}$. For pions its magnitude and phase are dominated by the $\rho$ meson peak in a broad mass region around $800 \mathrm{MeV}$.

\subsection{Interfering subprocesses and C-odd observables}

The $\gamma^{*} \gamma$ and bremsstrahlung processes interfere in the $\pi^{+} \pi^{-}$channel; this provides an opportunity to study the $\gamma^{*} \gamma$ contribution at amplitude level. Thanks to the different charge conjugation properties of the two processes their interference term can be selected by the charge asymmetries

$d \sigma\left(\pi^{+}(p) \pi^{-}\left(p^{\prime}\right)\right)-d \sigma\left(\pi^{-}(p) \pi^{+}\left(p^{\prime}\right)\right)$

Or

$d \sigma\left(e^{+} \gamma \rightarrow e^{+} \pi^{+} \pi^{-}\right)-d \sigma\left(e^{-} \gamma \rightarrow e^{-} \pi^{+} \pi^{-}\right)$,

while it drops out in the corresponding charge averages. We note that bremsstrahlung has an amplitude with both real and imaginary parts, especially at values of $W$ where it is dominated by vector meson resonances, and in particular benefits from the $\rho$ peak at $W$ around $800 \mathrm{MeV}$.

\subsection{Angular dependence}

The dependence of the $\gamma^{*} \gamma$ process on $\theta$ and $\varphi$ is entirely due to the decomposition of the $\pi \pi$ final state into partial waves, while in the limit $W^{2} \ll Q^{2}$ the interference contribution has an additional factor $\sin (\theta) \cos (\varphi)$ coming from the bremsstrahlung amplitude. This leads to very specific patterns which will help testing the helicity selection rules valid in the large- $Q^{2}$ limit, and thus the applicability of our theoretical description, at a finite value of $Q^{2}$. We will show elsewhere 14 these distributions with specific model assumptions for the generalized distribution amplitudes.

\section{UNIVERSALITY}

A new hadronic matrix element such as the generalized distribution amplitude is of great interest since it appears as a universal quantity in different processes. This is indeed the case as can be seen from an analysis of the electroproduction process $\gamma^{*} N \rightarrow \pi \pi N$ 9,15.

The amplitude for deep electroproduction of one meson $(\pi, \rho, \ldots)$ in the forward region has been shown to factorize [16] as the convolution of a hard scattering kernel $H$ and two nonperturbative objects obeying their own QCD evolution equations,

- a non-diagonal parton distribution [6] $f\left(x, x-x_{B}, t, \mu\right)$,

- the distribution amplitude of the produced meson $\phi(z, \mu)$,

as

$$
\begin{array}{r}
M=\int_{0}^{1} d z \int_{0}^{1} d x f\left(x, x-x_{B}, t, \mu\right) \\
\quad \times H\left(Q^{2}, x / x_{B}, z, \mu\right) \phi(z, \mu) .
\end{array}
$$

The introduction of generalized distribution amplitudes allows one to enlarge the scope of this factorization property to the case of nonresonant $\pi \pi$ production. This means that nonresonant $\pi \pi$ emission (including the $\pi^{0} \pi^{0}$ channel absent in $\rho$-decay) will have the same scaling behavior as $\rho$-production. Its amplitude is obtained in a straightforward way from $(13)$ by replacing $\phi \rightarrow \Phi$

\section{CONCLUSION}

To our knowledge no data exist as yet for exclusive two-meson production in $\gamma^{*} \gamma$-collisions in the kinematical domain where QCD factorization would enable us to extract generalized distribution amplitudes. An estimation of expected yields at various energies is under way 14. Promising experimental set-ups should be the high luminosity, medium energy $e^{+} e^{-}$colliders known as the B-factories at SLAC and KEK. The hadronic physics potential of these new machines is greatly enhanced by this new instance of factorization. 


\section{Acknowledgments}

We gratefully acknowledge many discussions with O. Teryaev. This work has been partially funded through the European TMR Contracts No. FMRX-CT96-0008: Hadronic Physics with High Energy Electromagnetic Probes and No. FMRX-CT98-0194: Quantum Chromodynamics and the Deep Structure of Elementary Particles. SUBATECH is Unité mixte 6457 de l'Université de Nantes, de l'Ecole des Mines de Nantes et de l'IN2P3/CNRS. Centre de Physique Théorique is Unité mixte C7644 du CNRS.

\section{REFERENCES}

1. M. Diehl, T. Gousset, B. Pire and O.V. Teryaev, Phys. Rev. Lett. 81, 1782 (1998).

2. D. Müller et al., Fort. Phys. 42, 101 (1994), hep-ph/9812448.

3. A. Freund, hep-ph 9903489.

4. S. Ong, Phys. Rev. D52, 3111 (1995);

R. Jakob, P. Kroll and M. Raulfs, J. Phys. G22, 45 (1996);

P. Kroll and M. Raulfs, Phys. Lett. B387, 848 (1996);

A.V. Radyushkin and R.T. Ruskov, Nucl. Phys. B481, 625 (1996);

I.V. Musatov and A.V. Radyushkin, Phys. Rev. D56, 2713 (1997).

5. CLEO Collab., J. Gronberg et al., Phys. Rev. D57, 33 (1998).

6. X. Ji, Phys. Rev. Lett. 78, 610 (1997);

A.V. Radyushkin, Phys. Rev. D56, 5524 (1997);

J.C. Collins and A. Freund, Phys. Rev. D59, 074009 (1999).

7. M. Diehl et al., Phys. Lett. B411, 193 (1997).

8. For a recent review and references, see M. Krawczyk, M. Staszel and A. Zembrzuski, hep-ph/9806291.

9. M.V. Polyakov, hep-ph/9809483.

10. M.V. Polyakov and C. Weiss, hepph/9902451.

11. G.P. Lepage and S.J. Brodsky, Phys. Lett. B87, 359 (1979);

A.V. Efremov and A.V. Radyushkin, Phys. Lett. B94, 245 (1980).
12. M.K. Chase, Nucl. Phys. B174, 109 (1980).

13. V.M. Budnev et al., Phys. Rept. C15, 181 (1975).

14. M. Diehl, T. Gousset and B. Pire, in preparation.

15. M. Diehl, T. Gousset and B. Pire, to appear in the Procs. of the Workshop on Exclusive and Semiexclusive Processes at High Momentum Transfer, Jefferson Lab, USA, 20-22 May 1999.

16. J.C. Collins, L. Frankfurt and M. Strikman, Phys. Rev. D56, 2982 (1997). 